\title{
Realization and swimming performance of the breaststroke by a swimming humanoid robot
}

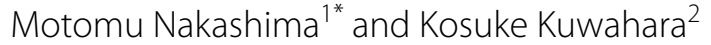

\begin{abstract}
In order to clarify the mechanics of human swimming, a full-body swimming humanoid robot called "SWUMANOID" was developed as an experimental platform for research about human swimming. SWUMANOID had a detailed human body shape, created using three-dimensional scanning and printing equipment, and was developed as an experimental model substituting for human subjects. Not only the appearance but also the methodology to realize various swimming strokes was considered. In order to reproduce complicated swimming motions with high fidelity, 20 waterproof actuators were installed. The free swimming of the crawl stroke at a velocity of $0.24 \mathrm{~m} / \mathrm{s}$ was realized in the previous study. However, it could not perform the breaststroke due to mechanical limitations. The objectives of this study were to realize the breaststroke for SWUMANOID by improving its lower limbs, and to investigate the swimming performance of the breaststroke experimentally. The lower body of SWUMANOID was fully redesigned, built, and connected to the upper body. The swimming motion of the breaststroke was created based on that of an actual swimmer. A free swimming experiment was conducted in a $25 \mathrm{~m}$ outdoor swimming pool. In addition, in order to discuss the experimental results in detail, the experiment was reproduced by the simulation. From the experiment, it was found that SWUMANOID could perform the breaststroke successfully. The swimming speed for the stroke cycle of $2.3 \mathrm{~s}$ was found to be $0.12 \mathrm{~m} / \mathrm{s}$. Since this swimming speed was considered low compared to that of the actual swimmer, the reason for the discrepancy was examined by simulation. From the simulation, it was found that one main reason for the low swimming speed was insufficient output power of the motors, especially for the knee and shoulder joints.
\end{abstract}

Keywords: Swimming, Sports engineering, Biomimetics, Breaststroke, Fluid forces, Humanoid robot

\section{Background}

In spite of such a long history of human swimming, its mechanics still have not been fully clarified since it is an extremely complicated phenomenon, in which a complex human body moves unsteadily with many degreesof-freedom (DOF) in the three-dimensional water flow. For example, the hand path in the crawl stroke depicts a distorted ellipse when viewed from the side in absolute space [1], showing that the hand does not push the water straight at a constant depth. Furthermore, the

\footnotetext{
*Correspondence: motomu@mei.titech.ac.jp

${ }^{1}$ Graduate School of Information Science and Engineering, Tokyo Institute of Technology, 2-12-1 Ookayama, Meguro, Tokyo 152-8552, Japan

Full list of author information is available at the end of the article
}

kinematics of the arm and hand during the underwater stroke is highly unsteady [2]. From this viewpoint, many attempts were made recently to quantify the unsteady fluid forces acting on a swimmer while swimming. The first approach was an experiment involving a human subject [3-5]. However, this method had problems with insufficient repeatability, physical fatigue of the subject, and difficulty in installing sensors on the subject. For this reason, some researchers have conducted experiments using physical models such as robots instead of human subjects. A lot of measuring experiments using physical models have been conducted to date [6-9], but there was no full-body experimental platform which could consider interactions between the many segments involved in normal swimming motions. Therefore, an analysis 
using physical models had been performed on an isolated segment and misleading conclusions could have been developed. To solve such problems, a full-body swimming humanoid robot was developed for research about human swimming by Chung and Nakashima [10]. The robot was named SWUMANOID. SWUMANOID had a detailed human-body shape, and was created using threedimensional scanning and printing equipment since it was developed for the experimental model substituting for human subjects. The size of SWUMANOID was $1 / 2$ scale of an actual swimmer. Not only the appearance but also the methodology to realize various swimming strokes was considered. In order to reproduce complicated swimming motions with high fidelity, 20 waterproofed actuators were installed. The free swimming of the crawl stroke at a velocity of $0.24 \mathrm{~m} / \mathrm{s}$ was realized in the previous study [11].

Developing a swimming humanoid robot such as SWUMANOID is important for the following two reasons. First, it is expected that it will become an experimental platform for the research of human swimming. To date, many simulation studies about human swimming have been conducted [12-15]. Such simulation technique is very useful and powerful tool for analysis. However, simulation always needs validation and improvement by comparing with experimental results, since it is not an actual phenomenon after all. In order to conduct more accurate experiments for that purpose, more elaborate physical models, such as swimming humanoid robots, will be necessary. The second reason for developing a swimming humanoid robot is that it can be applied to robots for special tasks accompanying water environment, such as rescue robots in the sea and working robots around a pool in a nuclear plant. Developing a swimming humanoid robot and studying how it can swim will be useful for developing such robots in the future.

SWUMANOID had sufficient DOF to perform not only the crawl stroke, but also the back and butterfly strokes. However, it could not perform the swimming motion of the breaststroke due to the following three reasons: (1) the thigh joint only had one DOF, (2) the knee joint could not be as fully flexed as a human's, (3) and the ankle joint could not be as fully dorsi-flexed as a human's as well. These points for the lower limbs did not become problems when SWUMANOID performed the flutter kick for the crawl stroke, but did when it performed the breaststroke. Indeed, for the breaststroke, the thigh joints have to possess three DOF since the legs move in a complicated manner. The knee and ankle joints also have to be fully flexed and dorsi-flexed, respectively, for the recovery position of the legs.

The objectives of this study were to realize the breaststroke for SWUMANOID by improving its lower limbs, and to investigate the swimming performance of the breaststroke experimentally. In this paper, the improvement for the breaststroke, creation of swimming motion, and experimental and simulation methods are explained. Next, the experimental results are shown and discussed. Finally, the obtained findings are summarized.

\section{Methods}

\section{Improvement for the breaststroke}

As mentioned above, SWUMANOID, which was developed in the previous study $[10,11]$, did not have sufficient DOF in the lower body for the breaststroke. Therefore, the lower body of SWUMANOID was completely redesigned for the present study. The overview and DOF configuration of the designed lower body are shown in Fig. 1. Hip joint 2 (yaw) and hip joint 3 (roll) were newly introduced. Since hip joint 2 was located in the same position as hip joint 1 , and there was no sufficient space for the motor, a belt pulley mechanism was introduced to this joint, as shown in Fig. 2a. The motor for this joint was installed in the thigh (the red part in Fig. 2a). With respect to the knee joint, the new joint had one degreeof-freedom, which was the same as the previously developed lower body. However, a much broader range of motion, especially in the flexing direction, was necessary for the breaststroke than for the crawl stroke. Therefore, the shank part was also re-designed to secure a sufficient flexion angle of $135^{\circ}$. For the ankle joint, a four-bar linkage mechanism was used, as shown in Fig. 2b. In particular, a large dorsi-flexion angle $\left(90^{\circ}\right)$ was secured to ensure the recovery position for the foot in the breaststroke. The outer cases of the robot were built by rapid prototyping

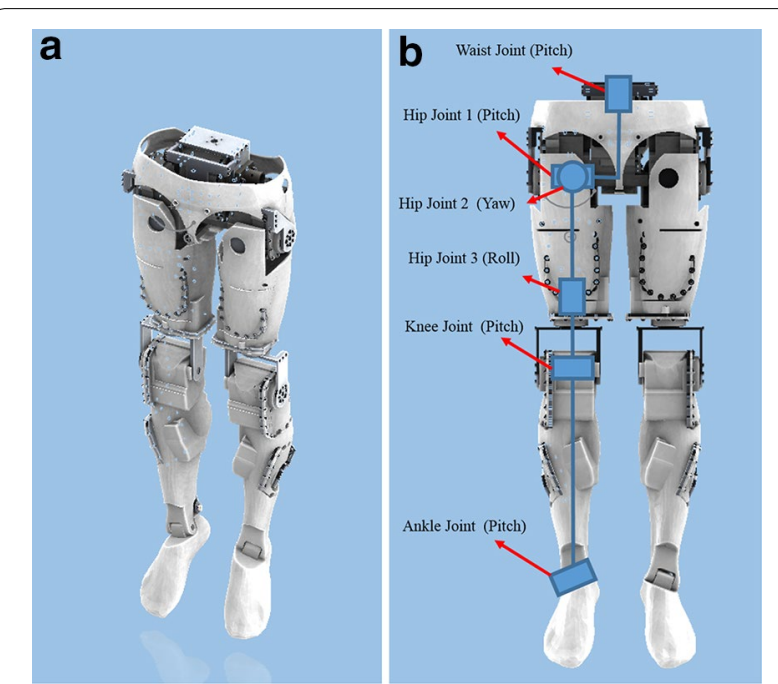

Fig. 1 Overview and DOF configuration of the designed lower body. a Overview, b DOF configuration 


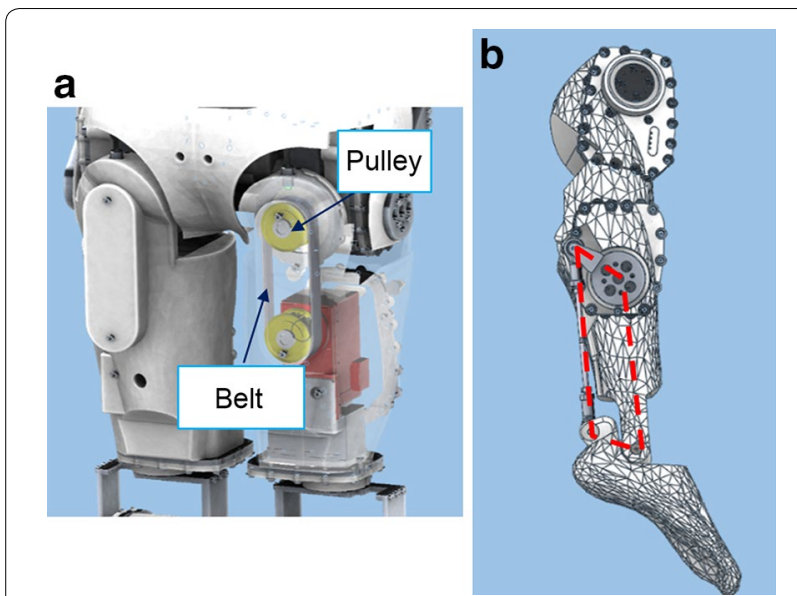

Fig. 2 Belt pulley mechanism for Hip joint 2 and four-bar linkage mechanism for the ankle joint. a Belt pulley mechanism, b four-bar linkage mechanism
Table 1 Specifications of improved SWUMANOID

\begin{tabular}{|c|c|}
\hline Items & Specifications \\
\hline Size & $\begin{array}{l}\text { (H) } 925 \mathrm{~mm}(\mathrm{~W}) 270 \mathrm{~mm}(\mathrm{D}) \\
119 \mathrm{~mm}\end{array}$ \\
\hline Weight & $7.4 \mathrm{~kg}$ \\
\hline $\begin{array}{l}\text { Actuators (dynamixel: Robotis } \\
\text { Corp.) }\end{array}$ & $\begin{array}{l}\mathrm{RX} 28: 2.5 \mathrm{Nm} \text { (at } 14.8 \mathrm{~V}) \\
\mathrm{M} 228: 3.1 \mathrm{Nm}(\text { at } 14.8 \mathrm{~V}) \\
\mathrm{M} 28: 7.3 \mathrm{Nm}(\text { at } 14.8 \mathrm{~V})\end{array}$ \\
\hline DOF & $\begin{array}{l}\text { Total: } 24 \text { DOFs } \\
\text { Arm: } 2 \text { arms } \times 6 \text { DOF } \\
\text { Waist: } 2 \text { DOF } \\
\text { Leg: } 2 \text { legs } \times 3 \text { DOF }\end{array}$ \\
\hline Controller & CM700 (Robotis Corp.) \\
\hline Battery & Li-Po 14.8 V 1550 mhA × 2 \\
\hline Communication & ZigBee module ZIG-110A \\
\hline
\end{tabular}

Dynamixel MX64 (Robotis inc., Seoul) was used for Waist joint, hip joint 1, hip joint 2, and knee joint, while dynamixel MX28 was used for hip joint 3 and ankle joint. CM700 (Robotis inc.) and ZIG-110A (Robotis inc.) were used for the controller of the robot and wireless communication

2, and knee joint, while dynamixel MX28 was used for hip joint 3 and ankle joint. For the controller of the robot and wireless communication, CM700 (Robotis inc.) and ZIG-110A (Robotis inc.) were used, respectively.

The parameters of the body segments are shown in Table 2. The weight and volume of each body segment were measured. In order to adjust the total buoyancy, weights were installed in the head and abdomen parts. As a result, the total specific gravity became slightly smaller than 1 , as shown in Table 2.

\section{Creation of swimming motion}

The overview of creating swimming motion is shown in Fig. 4. The swimming motion of the breaststroke in a previous simulation study by Nakashima [13] was used for the present study. This motion was created for the swimming human simulation model "SWUM" [12], based on the motion of an actual competitive swimmer. SWUM is a simulation model for analyses of human swimming, in which a swimmer's body is represented as a series of 21 truncated elliptic cones, and the rigid body dynamics and unsteady fluid forces are considered. By SWUM, it is possible to compute the fluid forces acting on a swimmer as well as joint torques, inputting the joint motion which is represented as multiple body positions in multiple time steps. This joint motion is defined using the original body reference coordinate system from SWUM, which is different from the relative coordinate system used for the robot. In addition, the arrangement of the joints and degrees-of-freedom for the robot and SWUM were different from each other. Therefore, the target position and orientation of body segments were first calculated in SWUM by forward kinematics. The joint angles of the
Fig. 3 Overview of the whole robot. The built lower body was connected to the previously developed upper body 
Table 2 Parameters of body segments

\begin{tabular}{lcccl}
\hline Segment & Weight [g] & $\begin{array}{l}\text { Added } \\
\text { weight [g] }\end{array}$ & $\begin{array}{l}\text { Volume } \\
{\left[\mathbf{c m}^{\mathbf{3}}\right]}\end{array}$ & $\begin{array}{l}\text { Specific } \\
\text { gravity }\end{array}$ \\
\hline Hip & 426 & & 410 & 1.04 \\
Thigh $\times 2$ & 1562 & & 1460 & 1.07 \\
Shank $\times 2$ & 954 & & 748 & 1.28 \\
Foot $\times 2$ & 106 & & 200 & 0.53 \\
Head and & 368 & 335 & 679 & 1.02 \\
$\quad$ neck & & & & \\
Upper & 660 & & 579 & 1.14 \\
$\quad$ arm $\times 2$ & & & 215 & 1.19 \\
Elbow $\times 2$ & 256 & & 306 & 0.98 \\
$\begin{array}{l}\text { Forearm } \times 2 \\
\text { Breast } \times 2\end{array}$ & 300 & & 1514 & 0.7 \\
$\begin{array}{l}\text { Waist } \\
\text { Whole upper }\end{array}$ & 3461 & 918 & 1460 & 0.97 \\
$\quad$ body & & & 4753 & 0.92 \\
Whole lower & 3048 & & 2818 & 1.08 \\
$\quad$ body & & & 7571 & 0.98 \\
Total & 7427 & &
\end{tabular}

The weight and volume of each body segment were measured. In order to adjust the total buoyancy, weights were installed in the head and abdomen parts. As a result, the total specific gravity became slightly smaller than 1

robot were then calculated by inverse kinematics, considering the motions of the scapular parts. The details of this procedure were described in the previous study [10]. Once the joint angles were obtained, they were put into CAD software, and the motions were checked visually. Finally, the motions were confirmed in a test on land. The obtained swimming motion of breaststroke displayed in the CAD software is shown in Fig. 5. The swimming motion was represented as 18 step motions in this case.

\section{Experimental method}

A free swimming experiment was conducted in a $25 \mathrm{~m}$ outdoor swimming pool. Two measuring tapes were placed on the poolside and on the water surface in the pool along with the swimming direction of the robot, as shown in Fig. 6. The swimming movement was filmed by two cameras, one on land and one in the water. From the images filmed by the camera on land, the swimming speed of the robot was calculated.

\section{Simulation method}

In order to discuss the experimental results in detail, the experiment was reproduced by the simulation using SWUM. The simulation model of SWUMANOID was constructed, as shown in Fig. 7. Since the body segments in SWUM were represented as truncated elliptic cones, the lengths and radii of the segments were determined based on the geometry of SWUMANOID.

\section{Results and discussion}

\section{Experimental results}

The swimming motion of SWUMANOID in the experiment is shown in Fig. 8. The stroke cycle was $2.3 \mathrm{~s}$ in this trial. This value was determined based on the stroke cycle of the actual swimmer, whose swimming motion was utilized for that of SWUMANOID in the present study,

\section{Analysis of actual swimming motion by a swimmer}

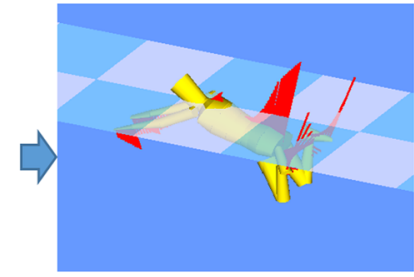

Step motion in SWUM

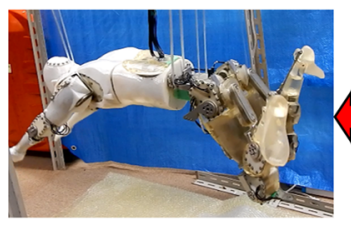

Confirmed in a test on land

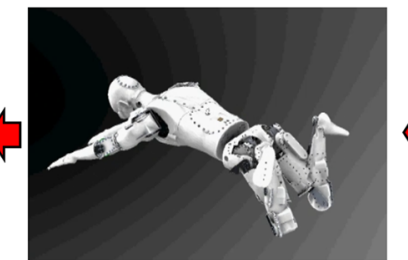

Checked by CAD software

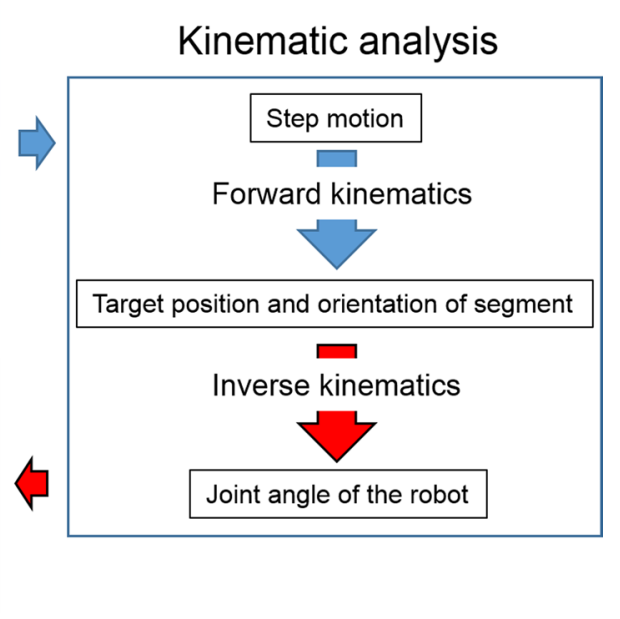

Fig. 4 Overview of creating the swimming motion. The target position and orientation of the body segments were first calculated in SWUM by forward kinematics. The joint angles of the robot were then calculated by inverse kinematics, considering the motions of the scapular parts. Once the joint angles were obtained, they were put into CAD software, and the motions were checked visually. Finally the motions were confirmed in a test on land 


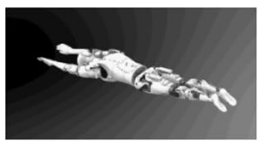

$1 / 18$

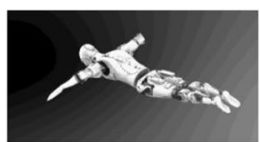

$7 / 18$

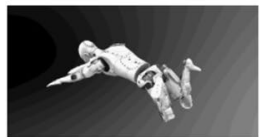

$13 / 18$

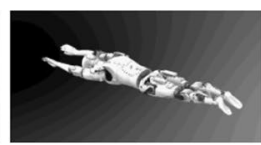

$2 / 18$

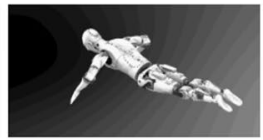

$8 / 18$

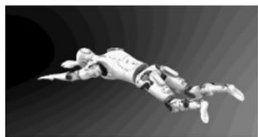

$14 / 18$

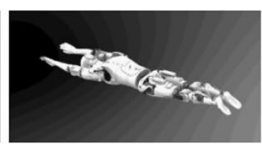

$3 / 18$

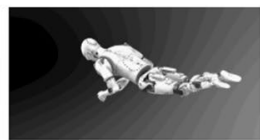

$9 / 18$

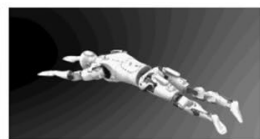

$15 / 18$

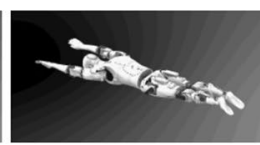

$4 / 18$

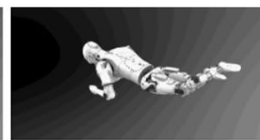

$10 / 18$

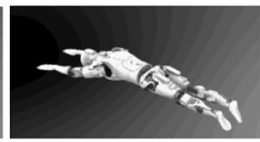

$16 / 18$

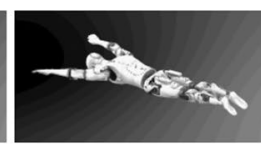

$5 / 18$

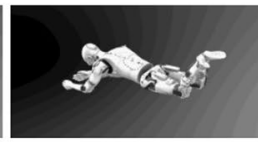

$11 / 18$

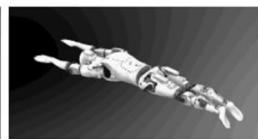

$17 / 18$

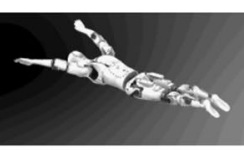

$6 / 18$

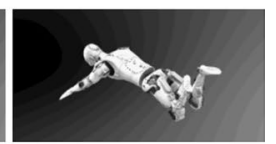

$12 / 18$

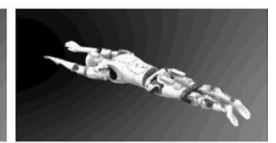

$18 / 18$

Fig. 5 Obtained swimming motion of breaststroke displayed in CAD software. The swimming motion was represented as an 18-step motion in this case

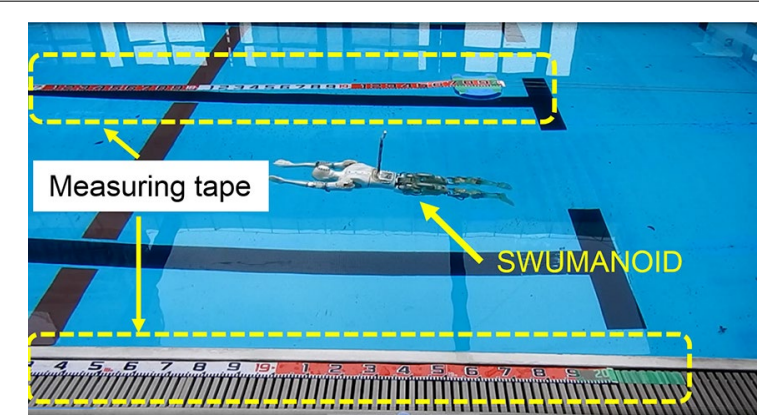

Fig. 6 Experimental setup. Two measuring tapes were placed on the poolside and on the water surface in the pool along with the swimming direction of the robot. The swimming movement was filmed by two cameras, one on land and one in the water. From the images filmed by the camera on land, the swimming speed of the robot was calculated

as well as the results of a preliminary experiment. In the preliminary experiment, the stroke cycle of $2.13 \mathrm{~s}$, which was the one for the actual swimmer, was tested as the first trial. However, it was found that the output power of the actuators were apparently insufficient so that the robot could not perform the programmed motion at all. Therefore the slightly longer stroke cycle of $2.3 \mathrm{~s}$ was chosen as the main trial. It was qualitatively confirmed that SWUMANOID could perform the breaststroke in the experiment successfully. The experimental result of swimming speed is shown in Fig. 9. Note that this result was the change of average speed in one stroke cycle. It was not possible to measure the instantaneous speed by the present experimental system due to lack of the accuracy. Since the fluctuation of the speed in one stroke cycle is seen in the actual breaststroke swimming and therefore is very important, it will be necessary to measure it by an improved experimental system in the future study. As shown in Fig. 9, the swimming speed almost converged to a constant value as time passed, and the average value was $0.12 \mathrm{~m} / \mathrm{s}$. The normalized stroke length, which is a non-dimensional propulsive distance in one stroke cycle and is defined by the product of the swimming speed and the stroke cycle divided by the stature, became 0.298 in this experiment. On the other hand, the normalized stroke length for the actual swimmer, whose swimming motion was utilized for that of SWUMANOID in the present study, was reported as 1.358 in the previous study [13] Note that the stroke cycle was $2.13 \mathrm{~s}$ in this case. It means that the swimming performance of SWUMANOID was almost one-fourth of that of an actual swimmer. One possible main reason of this discrepancy was the insufficient output power of the actuators. The target and measured values of the knee and shoulder joint angles for one stroke cycle are shown in Fig. 10. The shoulder joint angles are in the direction of flexion/extension. It was found that the measured angles (red) could not track the target angles (blue) both for the knee and shoulder when the angular velocities became large. The effect of this discrepancy in the joint angles is discussed by the simulation in the next section. Note that the measured angles for other joints were found to sufficiently track the target angles.

In addition, the trial of stroke cycle of $3.27 \mathrm{~s}$ was conducted. The average swimming speed was $0.11 \mathrm{~m} / \mathrm{s}$ in this case. This value was only slightly lower than $0.12 \mathrm{~m} / \mathrm{s}$ in the case of $2.3 \mathrm{~s}$. Conversely, $0.12 \mathrm{~m} / \mathrm{s}$ of $2.3 \mathrm{~s}$ was only slightly higher than $0.11 \mathrm{~m} / \mathrm{s}$ of $3.27 \mathrm{~s}$ although the stroke 

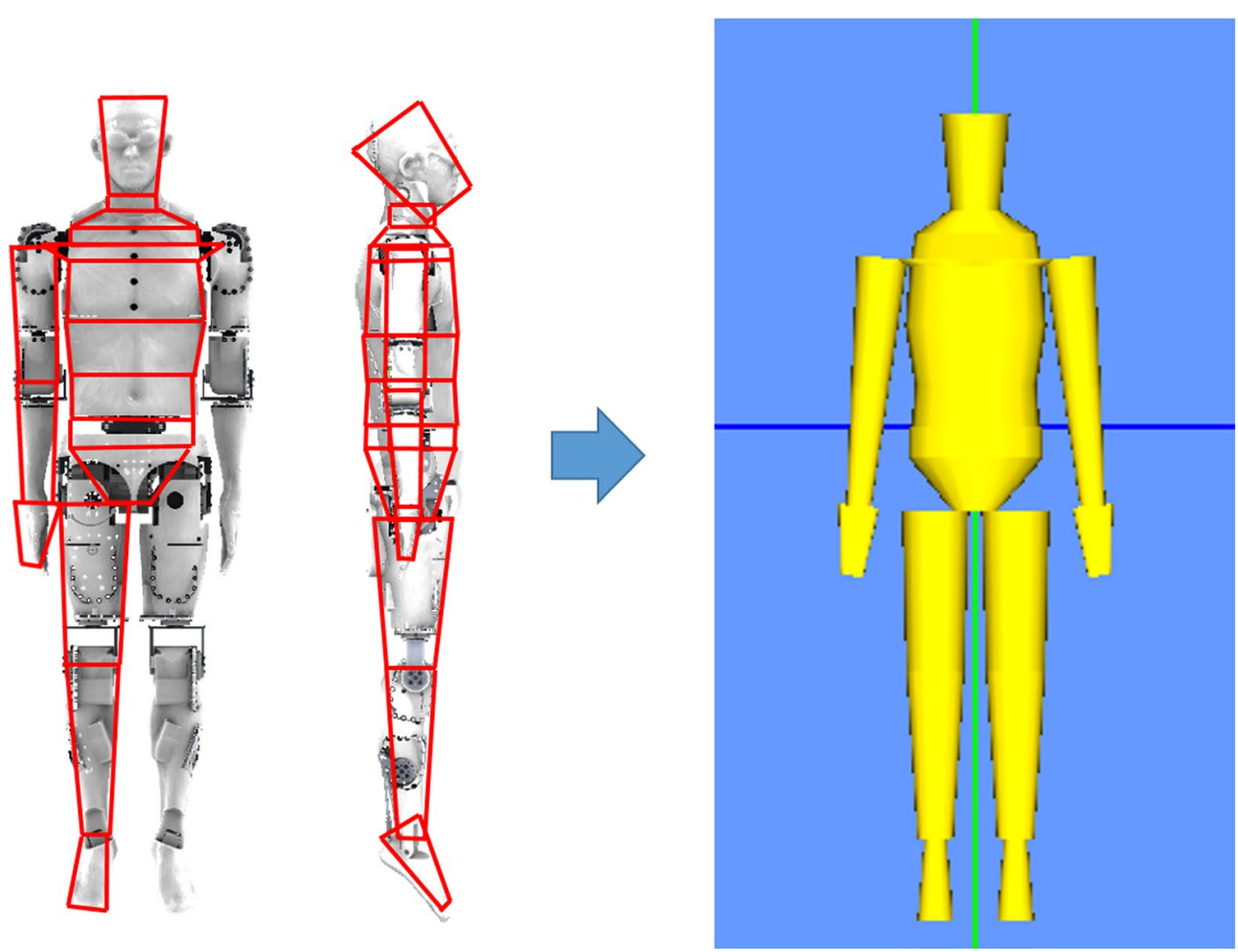

Fig. 7 Constructed simulation model of SWUMANOID. Since the body segments in SWUM were represented as truncated elliptic cones, the lengths and radii of the segments were determined based on the geometry of SWUMANOID

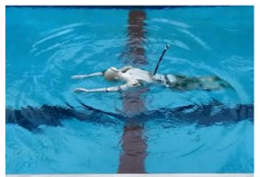

$0.00 \mathrm{~s}$

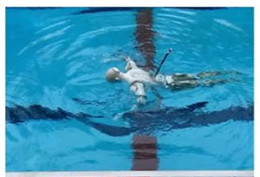

$0.77 \mathrm{~s}$

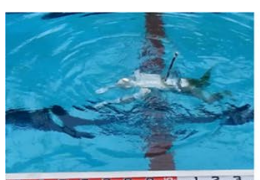

$1.53 \mathrm{~s}$

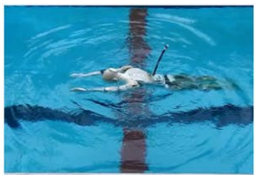

$0.13 \mathrm{~s}$

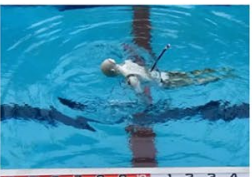

$0.89 \mathrm{~s}$

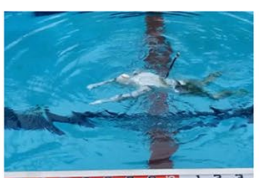

$1.66 \mathrm{~s}$

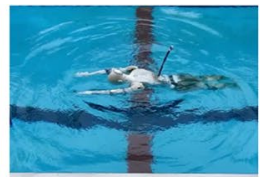

$0.26 \mathrm{~s}$

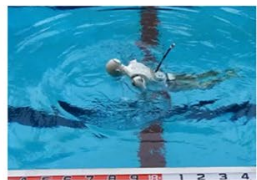

$1.02 \mathrm{~s}$

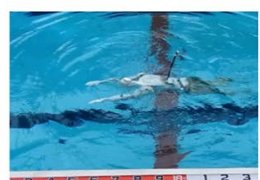

$1.79 \mathrm{~s}$

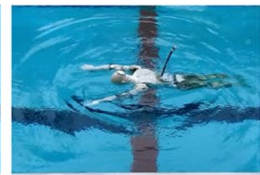

$0.38 \mathrm{~s}$

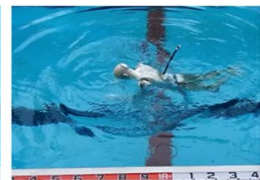

$1.15 \mathrm{~s}$

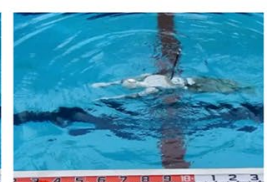

$1.92 \mathrm{~s}$

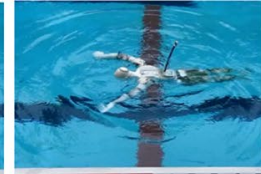

$0.51 \mathrm{~s}$

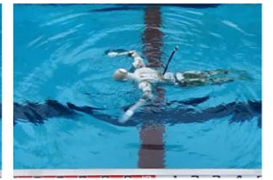

$0.63 \mathrm{~s}$

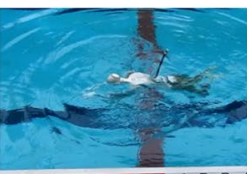

$1.28 \mathrm{~s}$

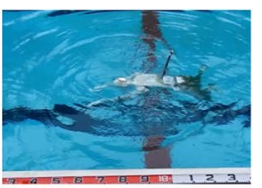

$1.41 \mathrm{~s}$

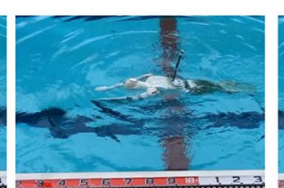

$2.04 \mathrm{~s}$

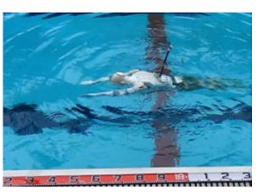

$2.17 \mathrm{~s}$

Fig. 8 Swimming motion of SWUMANOID in the experiment. The stroke cycle was $2.3 \mathrm{~s}$ in this trial. It was qualitatively confirmed that SWUMANOID could perform the breaststroke in the experiment as well 


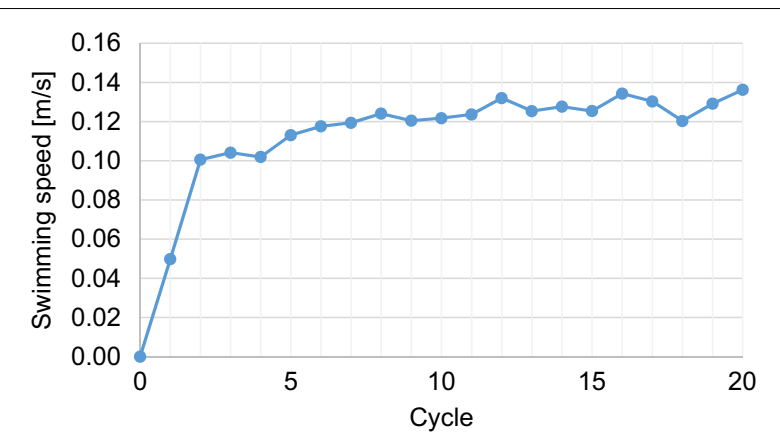

Fig. 9 Experimental result of swimming speed. The stroke cycle was $2.3 \mathrm{~s}$ in this trial. It almost converged to a constant value as time passed, and finally it reached $0.12 \mathrm{~m} / \mathrm{s}$ cycle became $70 \%$. Ideally, swimming speed is inversely proportional to stroke cycle. Therefore, from the result of $3.27 \mathrm{~s}$, the average swimming speed of $2.3 \mathrm{~s}$ was expected to be $0.11 / 0.7=0.15 \mathrm{~m} / \mathrm{s}$. Since $0.11 \mathrm{~m} / \mathrm{s}$ of $2.3 \mathrm{~s}$ in the experiment was certainly lower than $0.15 \mathrm{~m} / \mathrm{s}$, the insufficient output power of the actuators were suspected from this result as well.

\section{Examination by simulation}

In order to examine the effect of discrepancies in the joint angles, simulations in four conditions shown in Table 3 were carried out. In condition 1, the measured knee and shoulder joint angles were put into the simulation. That is, this simulation reproduced the experimental

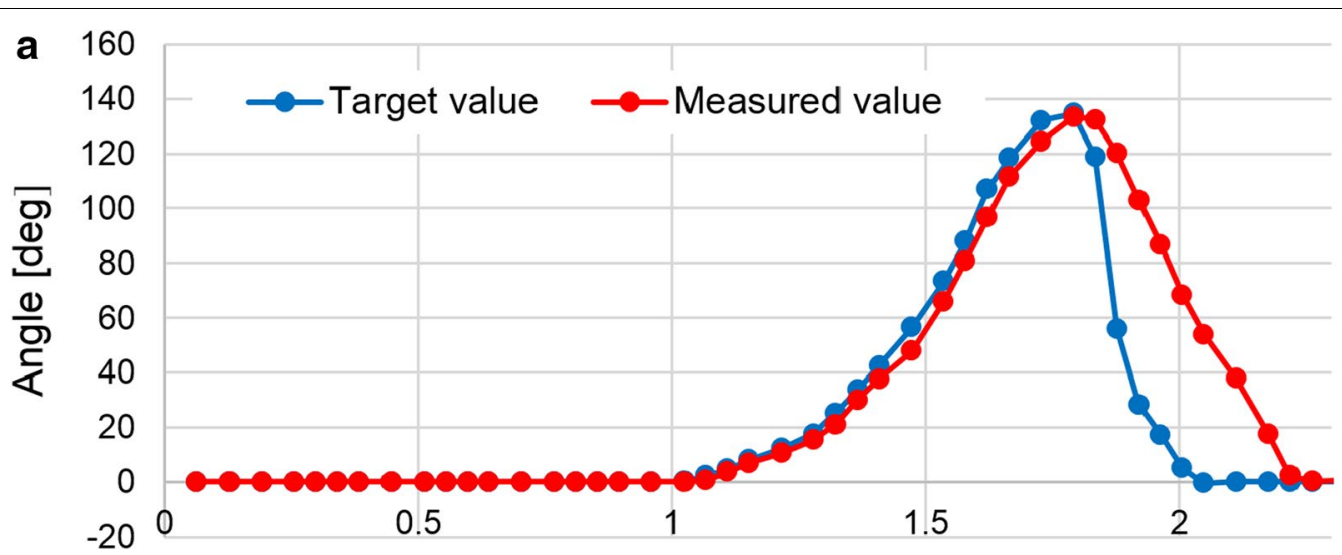

Time [s]

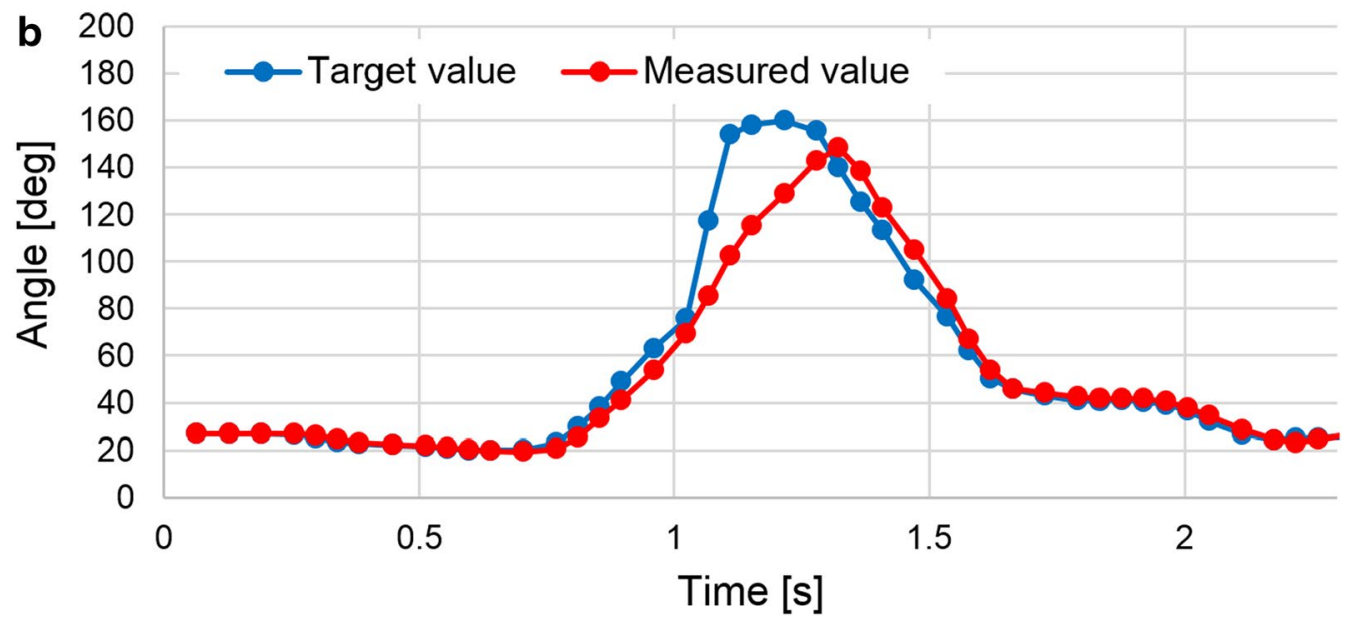

Fig. 10 Target and measured values of the knee and shoulder joint angles for one stroke cycle. a Knee joint angle, b shoulder joint angle. The shoulder joint angles are in the direction of flexion/extension. It was found that the measured angles could not track the target angles both for the knee and shoulder when the angular velocities became large 
Table 3 Simulation results of swimming speeds for four conditions

\begin{tabular}{llllc}
\hline Condition & Shoulder joints & Knee joints & Swimming speed [m/s] & Increase amount [\%] \\
\hline 1 & Measured & Measured & 0.118 & 0 \\
2 & Target & Measured & 0.162 & 37 \\
3 & Measured & Target & 0.166 & 41 \\
4 & Target & Target & 0.21 & 78
\end{tabular}

In condition 1, the measured knee and shoulder joint angles were put into the simulation. In condition 2, the target values of the joint angles for the shoulders were put into the simulation instead of the measured values. In condition 3, the target values for the knees were put into the simulation. In condition 4 , the target values both for the shoulders and knees were put into the simulation

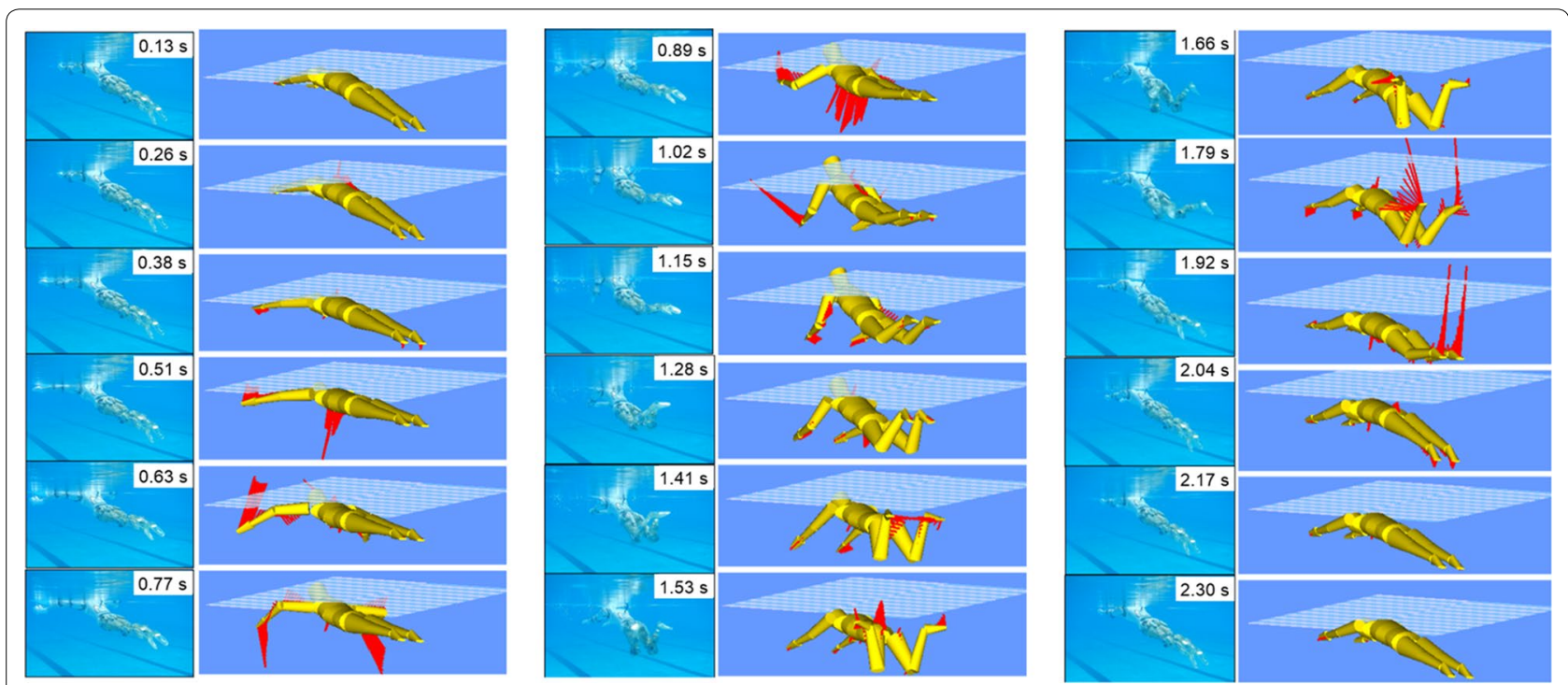

Fig. 11 Comparison of the underwater filmed images in the experiment and images in the simulation reproducing the experimental condition (condition 1). It was confirmed that the behavior of SWUMANOID in the simulation was consistent with that in the experiment

condition. Note that the target angles were put into the simulation for all the other joints since the measured angles for the other joints were found to sufficiently track the target angles. In condition 2, the target values of the joint angles for the shoulders were put into the simulation instead of the measured values. In condition 3 , the target values for the knees were put into the simulation. In condition 4 , the target values both for the shoulders and knees were put into the simulation. Comparison of the underwater filmed images in the experiment and images in the simulation reproducing the experimental condition (condition 1) is shown in Fig. 11. It was visually confirmed that the behavior of SWUMANOID in the simulation was consistent with that in the experiment. Note that one of the fluid force coefficients in the simulation model was adjusted so that the swimming speed in the simulation of condition 1 became equal to that in the experiment. The simulation results of swimming speeds for the four conditions are shown in Table 3. The increases in amounts of swimming speed against the condition 1 are also shown in the table. It was found that the swimming speed increased by 37 and $41 \%$ by changing the joint angles from the measured values to the target ones for the shoulders and knees, respectively. It was also found that the swimming speed increased for $78 \%$ by changing the joint angles both for the shoulders and knees. The fluid forces acting on the hand, forearm and foot for one stroke cycle in conditions 1 and 4 are shown in Fig. 12. From Fig. 12a, it was found that the fluid forces acting on the hand and forearm in condition 1 had two negative peaks. From Fig. 12b, it was found that the fluid forces acting on the foot in condition 1 did not have a sharp positive peak at $t^{*}=0.7$, unlike those in condition 4. These drawbacks in condition 1 resulted a swimming speed lower than that in condition 4.

The maximum joint torques of the shoulder and knee were calculated for condition 4 by the simulation. These were 0.84 and $2.37 \mathrm{Nm}$, respectively. Indeed, these were sufficiently smaller than 7.3 Nm in Table 1 for MX-64, which was used for the shoulder and knee joints. 


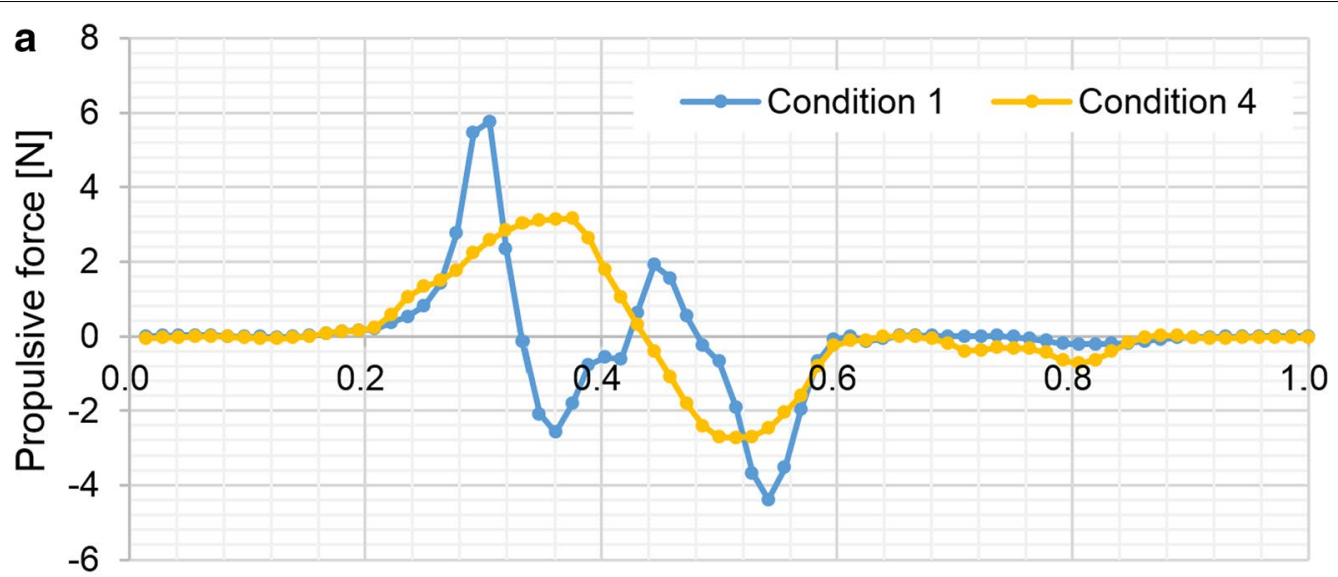

Non-dimensional time $t^{*}$

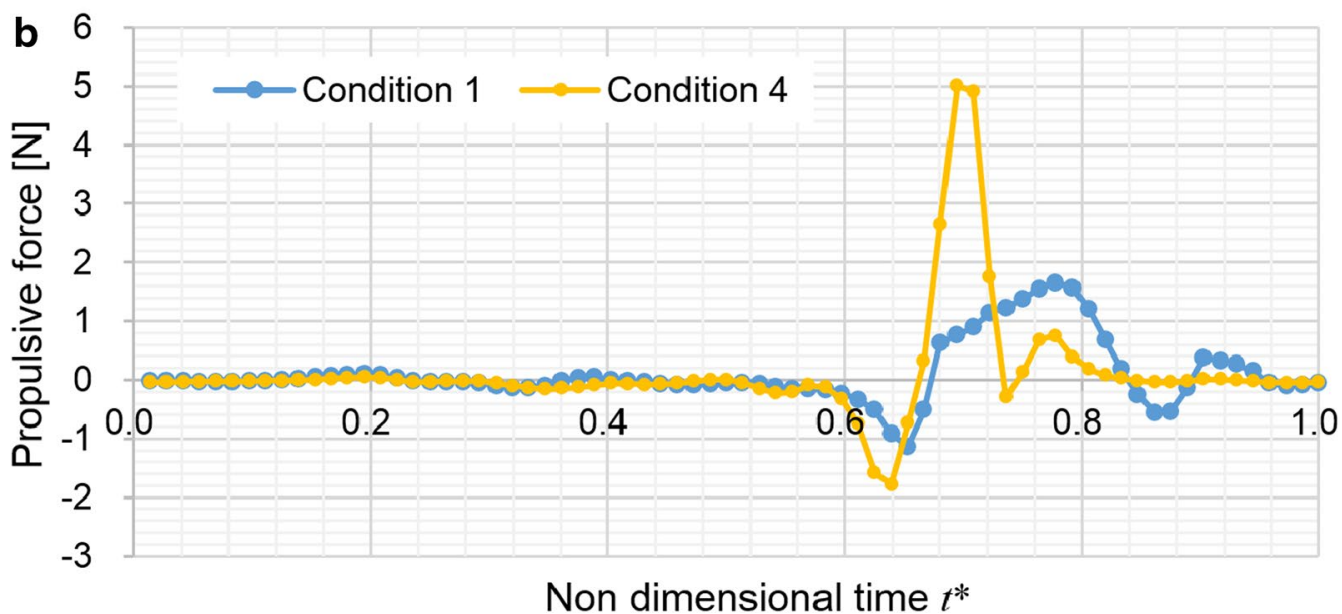

Fig. 12 Propulsive fluid forces acting on the hand, forearm, and foot for one stroke cycle in conditions 1 and 4. a Propulsive fluid forces acting on the right hand and forearm, $\mathbf{b}$ propulsive fluid forces acting on the right foot

However, 7.3 Nm was the stall torque, which was the maximum torque without rotation. It suggests that there is a possibility to improve the actuator performance by selecting more appropriate reduction ratio for the motor gears.

From above results, it was found that the insufficient output powers of the motors were one of the main reasons for the low swimming speed. Therefore, it is expected that the swimming speed will increase largely if the motors have sufficient output powers to realize the target joint motions. However, the swimming speed of $0.210 \mathrm{~m} / \mathrm{s}$ (condition 4) in Table 3 means the normalized stroke length of 0.522 . It is still much lower than 1.358 of an actual swimmer. One reason for this discrepancy may be that the difference in the actual joint angles and measured ones. The joint angles in the experiment were measured by the internal function of the motors, and therefore mechanical errors such as backlash of the joints were not taken into account. Therefore, it was possible that the actual joint angles had some differences from the measured values. Another possible reason is that the target joint angles themselves were not sufficiently well-considered. Although they were determined based on the actual values of an actual swimmer, they had to be modified for SWUMANOID due to the limitation of the degrees-offreedom as well as the range of motion of SWUMANOID. For example, in Fig. 12a, the fluid force acting on the hand and forearm still had a large negative peak at $t^{*}=0.5-0.6$. This means the recovery motion, in which the hand moves forward, still was not sufficiently good. If such problems in the swimming motion are all solved by modification, the swimming speed may increase more. 


\section{Conclusions}

In the present study, the swimming humanoid robot SWUMANOID was improved to perform the breaststroke. The lower body was fully redesigned, built and connected to the upper body. The swimming motion of the breaststroke was created based on that of an actual swimmer. From the experiment, it was found that SWUMANOID could perform the swimming motion of breaststroke successfully. The swimming speed for the stroke cycle of $2.3 \mathrm{~s}$ was found to be $0.12 \mathrm{~m} / \mathrm{s}$. From the examination by simulation, it was found that one of the main reasons for the low swimming speed was insufficient output power of the motors, especially for the knee and shoulder joints.

As the future tasks, the actuation system for the knee and shoulder has to be improved by some methods, such as providing instantaneous large current to the motors for the power peak timings, redesign of the motors or introducing subsidiary active/passive actuators.

\section{Authors' contributions}

MN provided the basic ideas of the overall system, and KK designed the robots and the overall system. All of the experiments were performed by MN and KK. All authors joined the discussions for this research. All authors read and approved the final manuscript.

\section{Author details}

${ }^{1}$ Graduate School of Information Science and Engineering, Tokyo Institute of Technology, 2-12-1 Ookayama, Meguro, Tokyo 152-8552, Japan. ${ }^{2}$ Graduate School of Science and Engineering, Tokyo Institute of Technology, 2-12-1 Ookayama, Meguro, Tokyo 152-8552, Japan.

\section{Acknowledgements}

This work was supported by JSPS KAKENHI Grant Number 26282174

\section{Competing interests}

The authors declare that they have no competing interests.

Received: 8 January 2016 Accepted: 11 April 2016

Published online: 26 April 2016
References

1. Maglischo EW (2003) Swimming fastest. Hum Kinet: 97

2. Toussaint HM, Van Den Berg C, Beek WJ (2002) Pumped-up propulsion during front crawl swimming. Med Sci Sports Exerc 34(2):314-319

3. Hollander AP, De Groot G, van Ingen Schenau GJ, Toussaint HM, De Best H, Peeters W, Meulemans A, Schreurs AW (1986) Measurement of active drag during crawl arm stroke swimming. J Sports Sci 4(1):21-30

4. Kolmogorov SV, Duplishcheva OA (1992) Active drag, useful mechanical power output and hydrodynamic force coefficient in different swimming strokes at maximal velocity. J Biomech 25(3):311-318

5. Takagi H, Sanders R (2002) Measurement of propulsion by the hand during competitive swimming. In: Ujihashi S, Haake SJ (eds) The engineering of sport 4. Blackwell Publishing, Oxford, pp 631-637

6. Lauder MA, Dabnichki P (2005) Estimating propulsive forces-sink or swim? J Biomech 38(10):1984-1990

7. Sidelnik NO, Young BW (2006) Optimising the freestyle swimming stroke: the effect of finger spread. Sports Eng 9(3):129-135

8. Nakashima M, Takahashi A (2012) Clarification of unsteady fluid forces acting on limbs in swimming using an underwater robot arm (development of an underwater robot arm and measurement of fluid forces). J Fluid Sci Tech 7(1):100-113

9. Nakashima M, Takahashi A (2012) Clarification of unsteady fluid forces acting on limbs in swimming using an underwater robot arm (2nd report, modeling of fluid force using experimental results). J Fluid Sci Tech 7(1):114-128

10. Chung C, Nakashima M (2013) Development of a swimming humanoid robot for research of human swimming. J Aero Aqua Bio-Mech 3(1):109-117

11. Chung C, Nakashima M (2013) Free swimming of the swimming humanoid robot for the crawl stroke. J Aero Aqua Bio-Mech 3(1):118-126

12. Nakashima M, Satou K, Miura Y (2007) Development of swimming human simulation model considering rigid body dynamics and unsteady fluid force for whole body. J Fluid Sci Tech 2(1):56-67

13. Nakashima M (2007) Analysis of breast, back and butterfly strokes by the swimming human simulation model SWUM. In: Kato N, Kamimura S (eds) Bio-mechanisms of swimming and flying -fluid dynamics, biomimetic robots, and sports science. Springer, Tokyo, pp 361-372

14. Nakashima M (2010) Modeling and simulation of human swimming. J Aero Aqua Bio-Mech 1(1):11-17

15. Takagi H, Nakashima M, Sato Y, Matsuuchi K, Sanders R (2015) Numerical and experimental investigations of human swimming motions. J Sports Sci. doi:10.1080/02640414.2015.1123284

\section{Submit your manuscript to a SpringerOpen ${ }^{\odot}$ journal and benefit from:}

- Convenient online submission

- Rigorous peer review

- Immediate publication on acceptance

- Open access: articles freely available online

- High visibility within the field

- Retaining the copyright to your article

Submit your next manuscript at springeropen.com 